\title{
A RAY TRACING SIMULATION OF SOUND DIFFRACTION BASED ON ANALYTIC SECONDARY SOURCE MODEL
}

\author{
Masashi Okada*, Takao Onoye* and Wataru Kobayashi** \\ * Graduate School of Info. Science and Tech., Osaka University, 1-5 Yamadaoka, Suita, Osaka, 565-0871, Japan \\ ** Arnis Sound Technologies, Co., Ltd., 2-7-9 Kitasenzoku, Ota-ku, Tokyo, 145-0062, Japan \\ email: \{okada.masashi,onoye\}@ist.osaka-u.ac.jp, kobachan@arns.com
}

\begin{abstract}
In this paper, we propose a novel method to estimate impulse response of sound diffraction based on ray tracing. This method is a Monte Carlo solution of multiple integration in analytic secondary source model of edge-diffraction, in which sample values of the integrand are calculated by ray tracing process. Similarity between our method and general ray tracing makes it possible to utilize various approaches developed for ray tracing. In our implementation, a ray tracing engine OptiX is employed, which exhibits good acceleration on a graphics processor. For more efficiency, we use importance sampling where insignificant diffraction paths are eliminated stochastically and thus reducing the number of samples. Based on these approaches, the proposed method achieves a processing performance of about $24 \mathrm{M}$ samples/sec for the simulation of first-order diffraction, which demonstrates the applicability to interactive simulation.
\end{abstract}

\section{INTRODUCTION}

Ray tracing is a popular technique to simulate wave propagation used in various fields such as $3 \mathrm{D}$ computer graphics (3DCG), electromagnetics and acoustics because of its computational simplicity. For example, acoustic analysis softwares such as Odeon [1] and CATT [2] use ray tracing. On the other hand, various acceleration and/or parallelization techniques have been proposed aiming at real-time ray tracing simulation in 3DCG field. Based on these techniques, interactive acoustic simulation has been getting more practical, which can enhance reality and immersion in virtual reality.

However, applying the general ray tracing techniques to acoustic simulation results in lack of diffraction which is significant especially in acoustics. For instance, acoustic simulation results described in [3] show that the lack of first- and higher-order diffractions causes undesired shadow of sound. In addition, subjective listening tests in [4] demonstrate that diffraction is also important not only in occluded zones but also in non-occluded zones.

There are several approaches to model edge-diffraction such as UTD (Uniform Theory of Diffraction) [3, 5] and Analytic Secondary Source Model (referred as the secondary source model) [6]. Although UTD can calculate edgediffraction more simply than the secondary source model, errors in low-frequency is significant [6] because UTD is a high-frequency approximation originally used in electromagnetics. On the other hand, the secondary source model can calculate edge-diffraction accurately based on the exact BiotTolstoy solution [7]. However, this model requires us to solve multiple integrations for high-order diffraction, which results in computational complexity.
In this paper, we focus on the secondary source model and its accuracy. Based on this model, we propose a novel method to estimate edge-diffraction impulse responses (IRs) using ray tracing. The number of diffraction paths evaluated in ray tracing is successfully reduced by importance sampling, i.e. a general technique for Monte Carlo integration. With the use of the state-of-the-art ray tracing engine running on a graphics processor, our method is capable of executing a sound diffraction simulation of a practical scene in real time.

\section{ANALYTIC SECONDARY SOURCE MODEL}

\subsection{Overview}

Let us consider a system illustrated in Fig. 1(a), comprising a sound source $S$, a receiver $R$ and an edge whose open angle is denoted as $\theta_{W}$. Here, $\left(r_{S}, \theta_{S}, z_{S}\right)$ and $\left(r_{R}, \theta_{R}, z_{R}\right)$ are cylindrical coordinates of $S$ and $R$, respectively. In this system, wave from $S$ is diffracted on the edge and then propagates to $R$. The diffractive IR observed at $R$ can be calculated by the exact Biot-Tolstoy solution [7]. Based on this solution, the secondary source model was derived [6], which provides a discrete Huygens interpretation of the solution. The interpretation is reviewed as follows.

First in Fig. 1(b), wave front from $S$ reaches an edge point $S^{\prime}$. Then $S^{\prime}$ is excited by the wave front and emits diffraction wave. After that, the wave reaches $R$. The above mentioned phenomenon arises at each edge point. Finally, the superposition of the diffraction waves from those points produces the Biot-Tolstoy's IR.

According to the secondary source model, the discrete time IR of the diffraction is calculated by the following equation, which is derived analytically from the Biot-Tolstoy solution,

$$
\begin{gathered}
h_{\mathrm{diffr}}[n]=\int_{\mathbb{R}_{n}}-\frac{v}{4 \pi} \frac{\beta\left[\alpha(z), \gamma(z), \theta_{S}, \theta_{R}\right]}{m(z) \cdot l(z)} d z \\
\forall z \in \mathbb{R}_{n}, n \leq \frac{m(z)+l(z)}{c} \cdot F_{S}<n+1,
\end{gathered}
$$

where $c$ is sound speed, $F_{S}$ is sampling frequency, $v$ is a parameter called wedge-index defined as $\pi / \theta_{W}$, and $\alpha, \gamma, m$ and $l$ are the path parameters of a diffraction wave illustrated in Fig. 1(b). $\beta[\cdot]$ is the directivity function of diffraction waves defined in next equation,

$$
\begin{gathered}
\beta\left[\alpha, \gamma, \theta_{S}, \theta_{R}\right]=\sum_{ \pm \pm} \frac{\sin \left[v\left(\pi \pm \theta_{S} \pm \theta_{R}\right)\right]}{\cosh [v \eta(\alpha, \gamma)]-\cos \left[v\left(\pi \pm \theta_{S} \pm \theta_{R}\right)\right]} \\
\eta(\alpha, \gamma)=\cosh ^{-1}\left(\frac{1+\sin (\alpha) \sin (\gamma)}{\cos (\alpha) \cos (\gamma)}\right)
\end{gathered}
$$




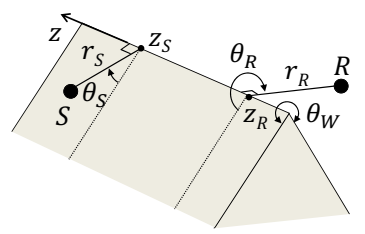

(a)

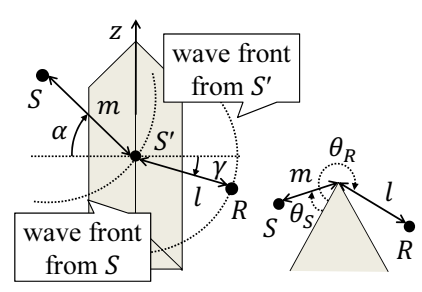

(b)
Figure 1: Illustration of first-order edge diffraction: (a) coordinate system (b) a discrete Huygens interpretation of diffraction according to the secondary source model.

From Eq. (1), we can find that the instantaneous value at $n$ is expressed as the function of path parameters of diffraction waves which arrive at $R$ at that time.

In this model, an edge is regarded as a new source. Based on this regard, contributions from the new edge are integrated to calculate high-order diffraction. Eq. (2) describes the formulation to calculate second-order diffraction illustrated in Fig. 2. $\beta_{1}$ and $\beta_{2}$ are diffraction factors of two edges, determined with the directivity function.

$$
\begin{array}{r}
h_{\mathrm{diffr}}[n]=\iint_{\mathbb{R}_{n}^{2}} \frac{v_{1} v_{2}}{(4 \pi)^{2}} \frac{\beta_{1}(\mathbf{z}) \cdot \beta_{2}(\mathbf{z})}{m_{1}(\mathbf{z}) \cdot l_{1}(\mathbf{z}) \cdot l_{2}(\mathbf{z})} d z_{1} d z_{2} \\
\forall \mathbf{z}=\forall\left(z_{1}, z_{2}\right) \in \mathbb{R}_{n}^{2}, n \leq \frac{m_{1}(\mathbf{z})+l_{1}(\mathbf{z})+l_{2}(\mathbf{z})}{c} \cdot F_{S}<n+1
\end{array}
$$

\subsection{Difficulties for Applications}

As indicated in the previous section, calculation of $K$ th-order diffraction needs to solve $K$ th-order integration. Since the integration cannot be solved analytically, general implementations of this model use the mid-point rule integration $[6,8,9]$. Based on this rule, Eq. (1) is approximated as

$$
\begin{gathered}
h_{\mathrm{diffr}}[n]=\sum_{i \in \mathbb{N}_{n}}\left(-\frac{v}{4 \pi} \frac{\beta\left[\alpha\left(z_{i}\right), \gamma\left(z_{i}\right), \theta_{S}, \theta_{R}\right]}{m\left(z_{i}\right) \cdot l\left(z_{i}\right)} \Delta z\right) \\
\forall i \in \mathbb{N}_{n}, n \leq \frac{m\left(z_{i}\right)+l\left(z_{i}\right)}{c} \cdot F_{S}<n+1,
\end{gathered}
$$

where $z_{i}$ is one of the mid-points in the equally subdivided regions.

Let $D$ be the number of divisions of an edge. Then this approximation needs to evaluate $D^{K}$ diffraction paths which grow exponentially in accordance with $K$. Therefore, much more computational cost is necessary for high-order diffraction. Although an adaptive edge-subdivision strategy has been proposed for fast calculation [10], this strategy is not applicable to high-order diffraction.

On the other hand, path validation process is needed for exact calculation. In this process, diffraction paths occluded by some kind of obstacles (e.g. walls) must be detected in order to eliminate the contributions of occluded waves. In a scene with many obstacles, the process may be complicated.

Consequently, computational costs of the integrations and/or the path validation make it difficult to calculate IRs with this model especially for high-order diffraction.

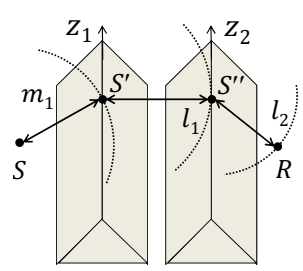

Figure 2: Interpretation of second-order diffraction. In practice, there are multiple diffraction path via all combination of $S^{\prime}$ and $S^{\prime \prime}$.

\section{PROPOSED METHOD}

Proposed method calculates the multiple integrations in the secondary source model by using Monte Carlo method [11]. Focusing on the integrand which is the function of diffraction paths parameters (i.e. propagation lengths and input/output angles), we use ray tracing to calculate an integrand's sample value (referred as $V_{S}$ ). In the ray tracing process, path validation is also conducted, in which $V_{S}$ is set to 0 so that the contribution of an occluded wave is eliminated.

\subsection{Procedure}

As input, our method requires information of a simulation scene: sound source $S$, receiver $R$ and an edge $E$. Figure 3 illustrates ray tracing procedure of the proposed method. First, (i) a ray is shot to $E$ from $S$ whereas $V_{S}$ is initialized to 1 . The intersection $S^{\prime}$ of the ray and $E$ (or the coordinate $z$ ) is determined randomly. If the ray intersects with $E$, (ii) a ray is shot to $R$ from $S^{\prime}$. At the same time, coefficients such as diffraction factor is multiplied to $V_{S}$. If the ray reaches $R$, (iii) $V_{S}$ is determined. In case that a ray does not reach $E$ or $R$, (ii) $/\left(\right.$ iii) ${ }^{\prime} V_{S}$ is set to 0 . For $K$ th-order diffraction $\left(S \rightarrow E_{1} \rightarrow \cdots \rightarrow E_{K} \rightarrow R\right), R$ is replaced by the next edge $E_{j}(j=2, \cdots, K)$ in (ii). The above mentioned procedure is repeated $M$ times, where $M$ is the number of evaluation paths (or the number of samples).

After the ray tracing process, IR estimation is conducted. Based on the average of the obtained sample values, the Monte Carlo estimates $h_{\mathrm{diffr}}[n]$ by the next equation,

$$
\begin{gathered}
h_{\mathrm{diffr}}[n] \simeq \frac{L_{1} \times \cdots \times L_{K}}{M} \sum_{i \in \mathbb{N}_{n}} V_{S, i} \\
\forall i \in \mathbb{N}_{n}, n \leq \frac{d_{i}}{c} \cdot F_{S}<n+1,
\end{gathered}
$$

where $i$ is the index of an evaluated path. $V_{S, i}$ and $d_{i}$ are the sample value and the total length of the path $i$, respectively. $L_{j}(j=1,2, \cdots, K)$ is the length of each edge.

\subsection{Features}

Considering that general ray tracing techniques are the Monte Carlo solutions of integral equation called rendering equation $[12,13]$, we can find that the objective and the procedure of the proposed method are similar to those of the general techniques. Because of this similarity, our method can be integrated into existing acoustic analysis software. Furthermore, various approaches dedicated to ray tracing can be applicable to our method.

Let $P$ be the number of primitives in a simulation scene. Then the computational cost of the proposed method is proportional to $K \times M \times P$. Differing from the mid-point rule, 


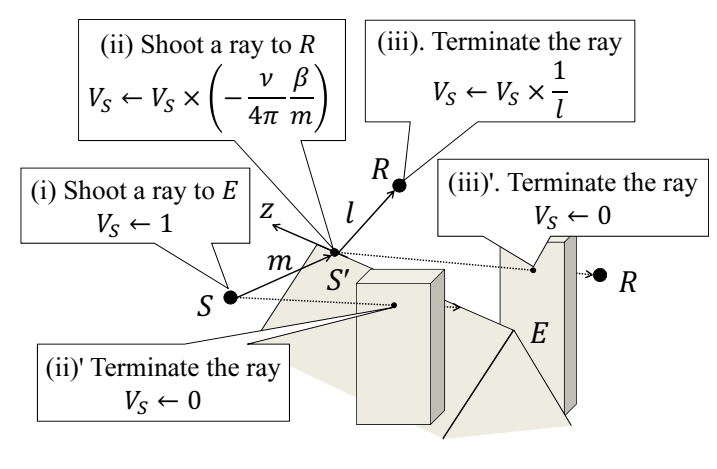

Figure 3: Procedure of ray tracing.

our method does not require the cost which increases exponentially with $K$. On the other hand, the term $M$ can be reduced with importance sampling introduced in Sect. 3.3. Furthermore, the term $P$, which arises from brute-force intersection detection for all primitives, can be reduced to $\log P$ by using so-called acceleration structures such as BVH (Bounding Volume Hierarchy) [14] and kd-tree [15].

\subsection{Importance Sampling}

In this section, importance sampling, which is a general technique for Monte Carlo integration [11], is introduced to reduce $M$ required for high-order simulation.

In case that integration variables $\mathbf{z}=\left(z_{1}, \cdots, z_{K}\right)$ are sampled based on probability density function (p.d.f.) $p(\mathbf{z})$, Eq. (3) is modified to

$$
h_{\mathrm{diffr}}[n] \simeq \frac{1}{M} \sum_{i \in \mathbb{N}_{n}} \frac{V_{S, i}}{p\left(\mathbf{z}_{i}\right)},
$$

where $\mathbf{z}_{i}$ is a set of the coordinate points on edges the path $i$ traced. The definition of a p.d.f. used in this paper is described below.

Let us consider the IR of first-order edge diffraction illustrated in Fig. 4(a). From this figure, we can notice that the IR attenuates rapidly after the onset. In other words, the contribution of the diffraction wave which propagates along a long path is insignificant. In order to eliminate such paths illustrated in Fig. 4(b), we introduce a p.d.f. $p_{c}(z)$ which is defined in the next equation,

$$
p_{c}(z)=\left[A w\left\{1+\left(\frac{z-z_{0}}{w}\right)^{2}\right\}\right]^{-1} .
$$

This is a modified Cauchy distribution whose range is limited to edge length $L . A$ is a normalization constant which is determined so that $\int_{L} p_{c}(z) d z=1$. Figure 5 illustrates the distribution and the geometric interpretation. In Fig. 5, a ray is shot from $S$ to $z$-axis with uniformly sampled launching angle $\theta$ then $z$ is Cauchy distributed. Based on this distribution, insignificant diffraction paths can be eliminated stochastically since the shorter a path is, the higher the sampling probability is, as shown in Fig. 5. For multiple integration, a binding distribution of $p_{c}(z)$ is used (i.e. $p(\mathbf{z})=p_{c}\left(z_{1}\right) p_{c}\left(z_{2}\right) ; K=2$ ).

\subsection{Discussion of Errors}

Let us consider estimation errors in Monte Carlo integration and the mid-point rule whose orders are respectively expressed as $O\left(N^{-1 / 2}\right)$ and $O\left(N^{-2 / K}\right)$, where $N\left(\equiv M=D^{K}\right)$

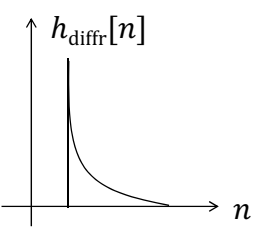

(a)

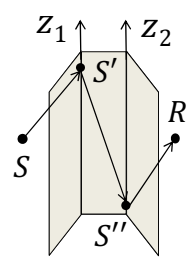

(b)
Figure 4: Examination of insignificant path: (a) IR of firstorder diffraction which attenuates rapidly after the onset (b) an example of long path (or insignificant path).

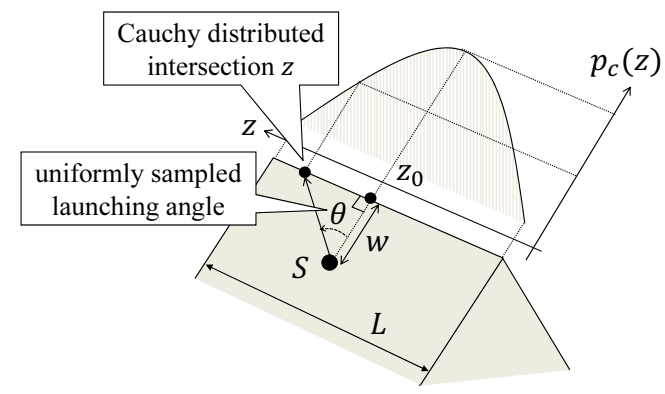

Figure 5: Cauchy distribution and the geometric interpretation. $w$ is length of the perpendicular line between $S$ and an edge. $z_{0}$ is the foot of the line.

is the number of evaluation paths. Therefore, the proposed method takes advantage for high-order diffraction (i.e. $K>$ 4 ) in terms of the error. Furthermore, the importance sampling can also compensate the disadvantage in case of $K \leq 4$, which is demonstrated later in Sect. 4.2.

\subsection{Implementation}

We implemented the proposed method using OptiX [16] which is a general purpose ray tracing engine distributed by NVIDIA. Owing to OptiX's parallelism utilizing a graphic processor, we have achieved fast implementation of our method. The graphics processor used in this research is GTX480.

\section{SIMULATION AND EVALUATION}

\subsection{Validation and Processing Time Evaluation}

First, we conduct a test to evaluate simulation accuracy of our method. Figure 6 illustrates a concert hall scene used in this test. The scene consists of $P=40$ primitives (or triangle meshes) and 39 diffraction edges whose open angles are $\theta_{W}<\pi$. For each edge, first-order diffraction is simulated. In this test, other wave phenomena (i.e. specular reflection and diffusion from walls) are not included. The number of samples assigned to each edge is 2,000 and thus totally $M=78,000(=39 \times 2,000)$ samples are evaluated. In order to validate the results, the estimated IR is compared with reference calculated by Edge diffraction toolbox for Matlab [9] which uses Simpson's rule (for $K=1$ ) and the mid-point rule (for $K>1$ ).

Figure 7 shows errors between the reference and the estimated IR. The IR is partially shown in Fig. 8, in which the errors are relatively compared with the IR. From Fig. 7, we can find that the estimated IR contains fleetly fluctuating noise. 


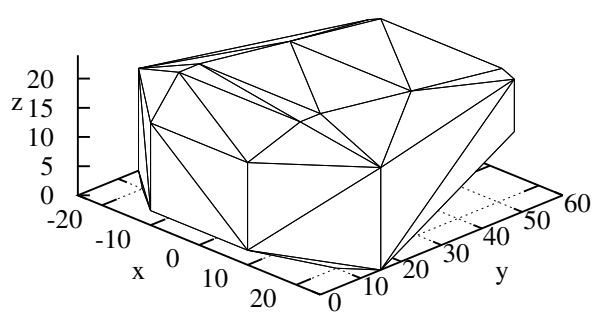

Figure 6: Simulation scene in Sect. 4.1 where $S:(-7.5,5,3)$, $R:(5,35,5)$. The details of this model can be found in [17].

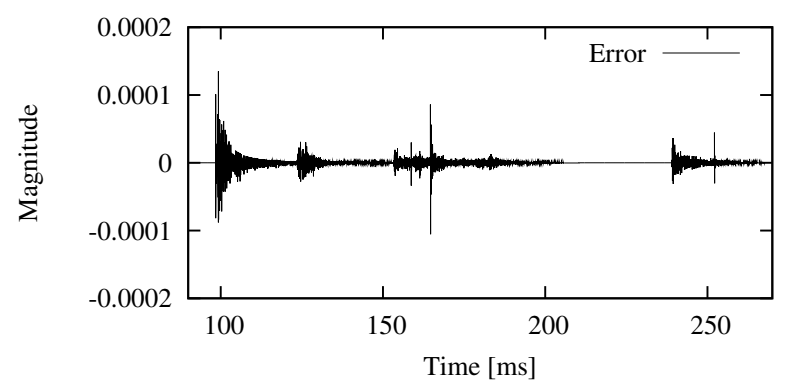

Figure 7: Errors between IRs estimated by Edge diffraction toolbox and the proposed method.

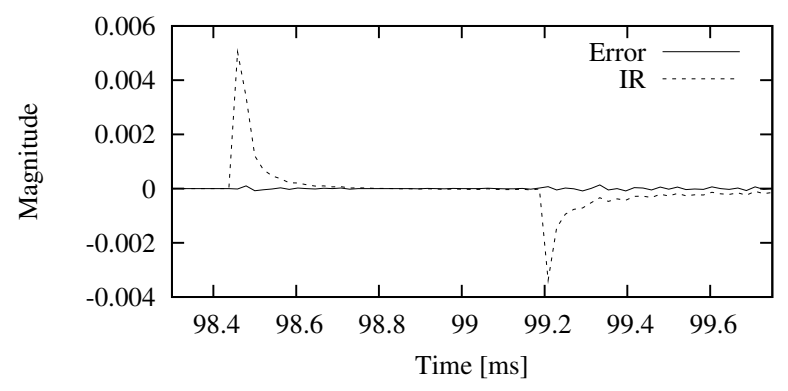

Figure 8: Comparison of errors and estimated IR focusing on the beginning of the IR. Root mean squared error normalized by the range of the reference value is $-62.6[\mathrm{~dB}]$.

Howerver the noise is sufficiently smaller than IR values as indicated in Fig. 8. From these results, we confirmed that the proposed method adequately estimates diffractive IRs.

We also evaluate the processing time of our method with varying $M \mathrm{~s}$. Figure 9 summarizes the evaluation result, from which we can confirm that the processing time of the above simulation (i.e. $M=78,000$ ) is approximately $4 \mathrm{~ms}$. Even in case with much larger samples $M \simeq 500,000$, the processing time is about $21 \mathrm{~ms}$ and thus our method achieves a processing performance of about $24 \mathrm{M}$ samples/sec. In terms of update rate in dynamic scenes, the rate approximates $50 \mathrm{~Hz}$ in the case of $M \simeq 500,000$. Based on these performance results, we can say that the method is sufficiently applicable to interactive simulation.

\subsection{Evaluation of Importance Sampling}

In this section, we conduct a test to evaluate the effectiveness of the importance sampling (IS). Figure 10 shows the simulation scene, where two diffraction edges are adjacently

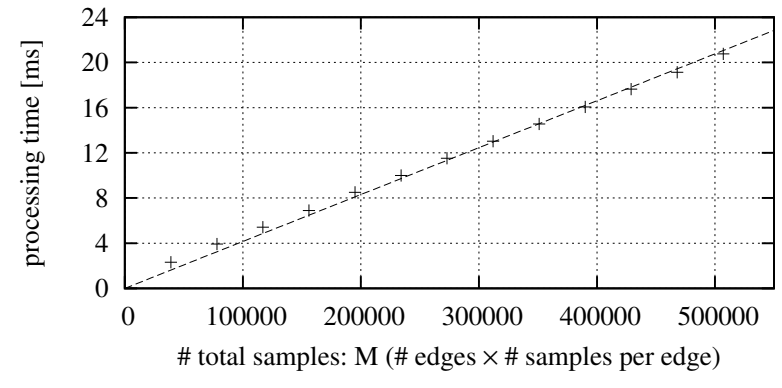

Figure 9: Number of samples vs. processing times required to simulate first-order diffraction in Fig. 6.

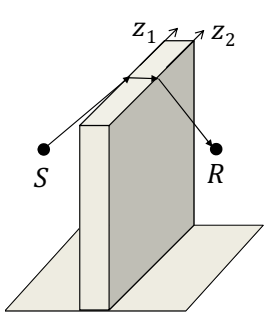

(a) perspective view

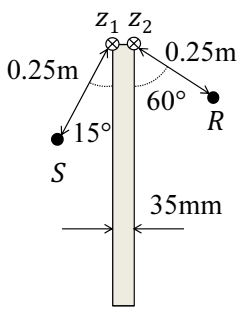

(b) side view

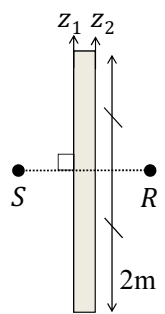

(c) top view
Figure 10: Simulation scene of second-order diffraction in Sect. 4.2. The similar scene is originally used in [6].

arranged. In this test, we simulate second-order diffraction with the proposed method where IS is enabled or disabled. The number of samples $M$ is set to $900,10,000$ and $1,000,000$. For comparison, estimation using the mid-point rule is also conducted. The number of edge division $D$ is set to 30,100 and 1,000 so that the number of evaluation paths $D^{2}$ is equal to $M$.

Figures 11 through 13 respectively show the estimation results of the proposed method (IS is disabled/enabled) and the mid-point rule. In these figures, arrival time of the diffraction wave from the shortest path is regarded as 0 . Although the IRs of $M=10,000$ are noisy in Figs. 11 and 13, the IR in Fig. 12 is sufficiently converged even though the same number of paths are evaluated. Furthermore in Fig. 12, the proposed method obtains IR converged much better than the others with only $M=900$ samples. From these results, we can confirm the following effectiveness described in Sects. 3.3 and 3.4; (1) the number of samples required for well converged results can be reduced based on IS, (2) in terms of the errors, the proposed method with IS can obtain better results than the mid-point rule if finite samples are available.

\section{CONCLUSION}

In this paper, we proposed an estimation method for edgediffraction IRs based on ray tracing. This method is a Monte Carlo solution of multiple integration in the secondary source model, where sample values calculation of integrand and path validation are conducted in ray tracing process.

Focusing on the procedures' similarity with general ray tracing, we implemented the proposed method by using a ray tracing engine OptiX. Consequently fast implementation has been achieved. In our test, we demonstrated that the processing time required to evaluate $M \simeq 500,000$ samples is about 


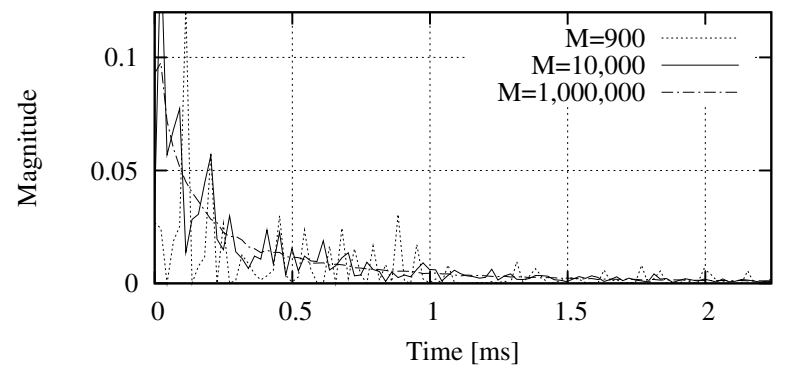

Figure 11: IR estimated by the proposed method (IS: disabled).

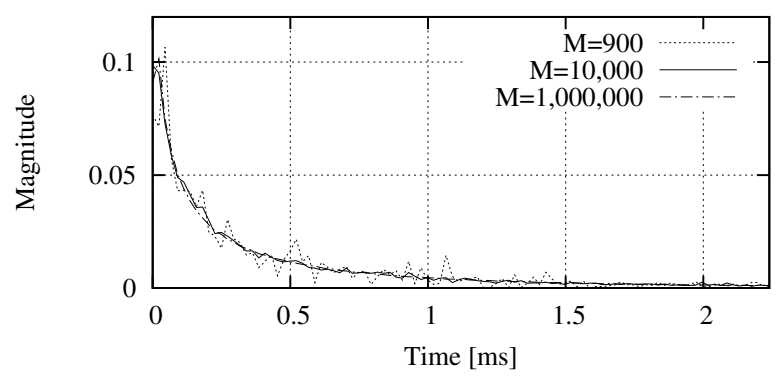

Figure 12: IR estimated by the proposed method (IS: enabled).

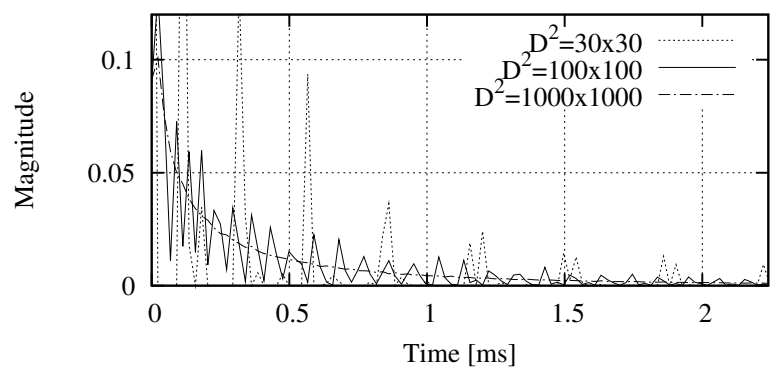

Figure 13: IR estimated by the mid-point rule.

$21 \mathrm{~ms}$, which indicates the applicability to interactive simulation. For more efficiency, importance sampling was introduced where insignificant diffraction paths are eliminated stochastically.

As for our future work, we will try to apply adaptive importance sampling strategies used in 3DCG field such as Metropolis Light Transport [18] and Photon Mapping [19] in order to make our method more flexible for any complex scenes. We also need to conduct detailed evaluation of estimated IRs' qualities, including subjective listening test.

\section{Acknowledgment}

This research has been supported by the Global COE program of the Ministry of Education, Culture, Sports, Science and Technology, Japan, under the title "Founding Ambient Information Society Infrastructure."

\section{REFERENCES}

[1] Odeon A/S Inc. Odeon Room Acoustics Software for prediction and auralisation. http://www.odeon.dk/.
[2] CATT Inc. CATT-Acoustic v8.0. http://www.catt.se/.

[3] N. Tsingos, T. Funkhouser, A. Ngan, and I. Carlbom. Modeling acoustics in virtual environments using the uniform theory of diffraction. In Proceedings of the 28 th annual conference on Computer graphics and interactive techniques, page 552, 2001.

[4] R. Torres, U. Svensson, and M. Kleiner. Computation of edge diffraction for more accurate room acoustics auralization. The Journal of the Acoustical Society of America, 109:600, 2001.

[5] R. Kouyoumjian and P. Pathak. A uniform geometrical theory of diffraction for an edge in a perfectly conducting surface. Proceedings of the IEEE, 62(11):14481461, 1974.

[6] U. Svensson, R. Fred, and J. Vanderkooy. An analytic secondary source model of edge diffraction impulse responses. The Journal of the Acoustical Society of America, 106:2331, 1999.

[7] M. Biot and I. Tolstoy. Formulation of wave propagation in infinite media by normal coordinates with an application to diffraction. J. Acoust. Soc. Am, 29(3):381391, 1957.

[8] T. Lokki, U. Svensson, and L. Savioja. An efficient auralization of edge diffraction. In AES 21st Int. Conf. on Architectural Acoustics and Sound Reinforcement, pages 166-172, 2002.

[9] U. Svensson. Edge diffraction toolbox for Matlab. http://www.iet.ntnu.no/ svensson/software/index.html.

[10] P. Calamia and U. Svensson. Fast time-domain edgediffraction calculations for interactive acoustic simulations. EURASIP Journal on Applied Signal Processing, 2007(1):186, 2007.

[11] W. Press, B. Flannery, S. Teukolsky, W. Vetterling, et al. Numerical recipes, volume 3. Cambridge university press Cambridge, 2007.

[12] J. T. Kajiya. The rendering equation. SIGGRAPH Computer Graphics., 20(4):143-150, 1986.

[13] S. Siltanen, T. Lokki, S. Kiminki, and L. Savioja. The room acoustic rendering equation. The Journal of the Acoustical Society of America, 122:1624-1635, 2007.

[14] T. Kay and J. Kajiya. Ray tracing complex scenes. ACM SIGGRAPH Computer Graphics, 20(4):269-278, 1986.

[15] T. Foley and J. Sugerman. KD-tree acceleration structures for a GPU raytracer. In Proceedings of the ACM SIGGRAPH EUROGRAPHICS conference on Graphics hardware, pages 15-22, 2005.

[16] S. Parker, J. Bigler, A. Dietrich, H. Friedrich, J. Hoberock, D. Luebke, D. McAllister, M. McGuire, K. Morley, A. Robison, et al. OptiX: a general purpose ray tracing engine. ACM Transactions on Graphics (TOG), 29(4):1-13, 2010.

[17] F. Mechel. Improved mirror source method in room acoustics. Journal of Sound and Vibration, 256(5):873940, 2002.

[18] E. Veach and L. Guibas. Metropolis light transport. In Proceedings of the 24th annual conference on Computer graphics and interactive techniques, pages 6576. ACM Press/Addison-Wesley Publishing Co., 1997.

[19] H. Jensen. Realistic image synthesis using photon mapping. AK Peters, Ltd. Natick, MA, USA, 2009. 